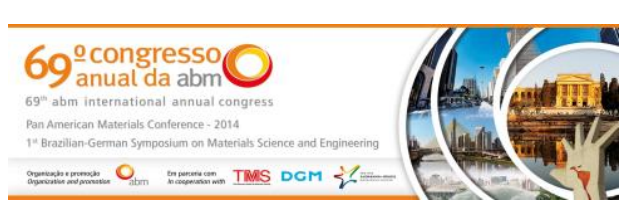

Tema: Tratamento Térmico

\title{
EFEITO DAS CONDIÇÕES DE TÊMPERA NA MICROESTRUTURA E NA DUREZA DO AÇO AISI M2*
}

\author{
Aline Silva Magalhães ${ }^{1}$ \\ Graziele Gianini Braga Maria ${ }^{2}$ \\ Suzanny Cristina Soares Martins ${ }^{3}$ \\ Tâmisa Eleutério Silva ${ }^{4}$ \\ Wellington Lopes ${ }^{5}$ \\ Elaine Carballo Siqueira Corrêa ${ }^{6}$
}

\section{Resumo}

Os aços rápidos são assim chamados por sua capacidade de reter elevadas durezas mesmo quando submetidos a altas temperaturas, em geral decorrentes de elevadas velocidades de corte no processo de usinagem. No entanto, para que esses aços apresentem as propriedades mencionadas, faz-se necessária a combinação adequada da composição química e dos parâmetros dos tratamentos térmicos. Dentro deste contexto, o objetivo do trabalho é avaliar os efeitos da temperatura de austenitização e do tempo de encharque na têmpera na microestrutura e na dureza do aço rápido AISI M2, ligado ao tungstênio e ao molibdênio. As análises foram conduzidas através de testes de dureza e microscopias óptica e eletrônica de varredura, além de difração por raios- $x$, considerando três temperaturas e três tempos de austenitização. Foi observado que, de maneira geral, os valores de dureza aumentaram com a elevação da temperatura de austenitização para os três diferentes tempos de encharque utilizados, de forma concomitante com a redução na quantidade de carbonetos dispersos na matriz martensítica.

Palavras-chave: Aço rápido; Tratamento térmico; Microestrutura; Dureza.

\section{EFFECT OF QUENCHING PARAMETERS IN THE MICROSTRUCTURE AND THE HARDNESS OF AISI M2 HIGH SPEED STEEL}

\section{Abstract}

The high speed steels are known for their ability to retain high hardness values even when subjected to high temperatures, usually resulting from high shear rates in the machining process. However, for the high speed steel having the aforementioned properties, it is necessary the combination of adequate chemical composition and heat treatment parameters. The aim of this work is to evaluate the effects of austenitizing temperature and soaking time in quenching in the microstructure and hardness of the AISI M2 high speed steel, whose chemical composition envolves tungsten and molybdenum. The analyzes were conducted by in terms of hardness and optical and scanning electronic microscopy. Three austenitizing temperatures and three soaking times were considered. It was observed that, in general, hardness values increased with austenitizing temperature, as well as the amount of carbides dispersed in the martensitic matrix diminished.

Keywords: High speed steel; Heat treatment; Microstructure; Hardness.

Eng. Materiais, Mestranda Eng. Materiais, CEFET-MG, Belo Horizonte, MG, Brasil

Eng. Materiais, Mestranda Eng. Materiais, CEFET-MG, Belo Horizonte, MG, Brasil

Eng. Materiais, Mestranda Eng. Materiais, CEFET-MG, Belo Horizonte, MG, Brasil

Eng. Materiais, Mestranda Eng. Materiais, CEFET-MG, Belo Horizonte, MG, Brasil

Eng. Mecânico, Dr., Professor, Depto. Eng. Materiais, CEFET-MG, Belo Horizonte, MG, Brasil

Eng. Mecânica, Dra., Professora, Depto. Eng. Materiais, CEFET-MG, Belo Horizonte, MG, Brasil

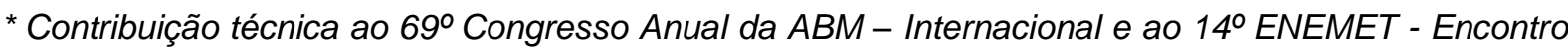
Nacional de Estudantes de Engenharia Metalúrgica, de Materiais e de Minas, 21 a 25 de julho de 2014, São Paulo, SP, Brasil. 


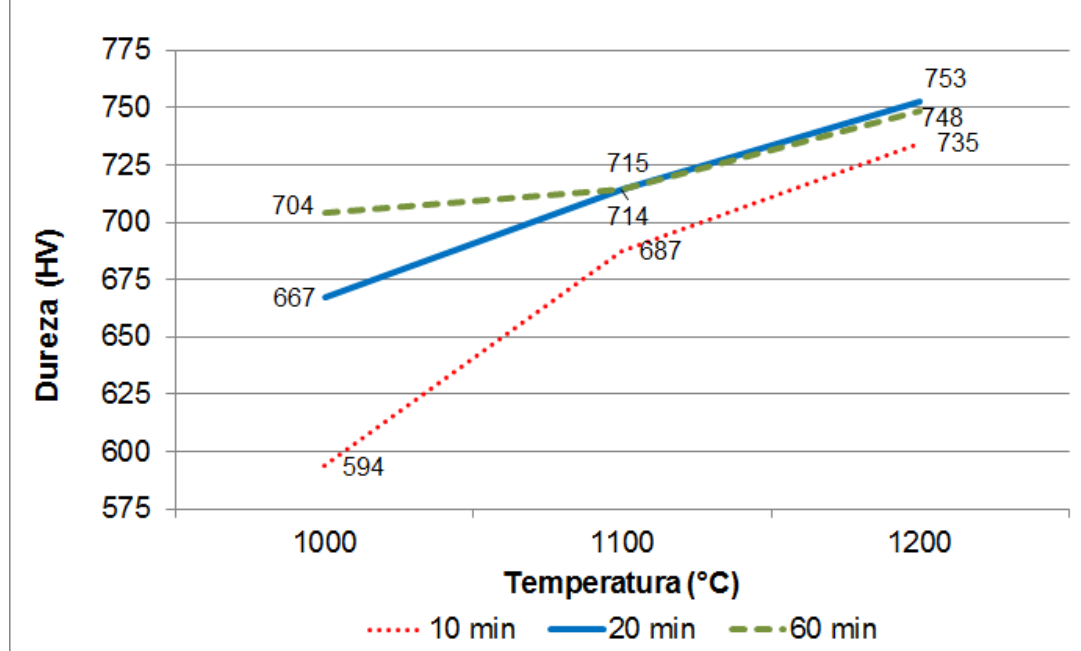

Figura 1. Gráfico comparativo das durezas apresentadas das amostras resfriadas em óleo relacionando a temperatura e o tempo de encharque

Nas Figuras 2, 3 e 4 podem ser observadas as fotomicrografias obtidas por microscopia ótica (MO) e microscopia eletrônica de varredura (MEV) do aço AISI M2 desenvolvidas após as diversas condições de tratamento térmico aplicado (Tabela 2).
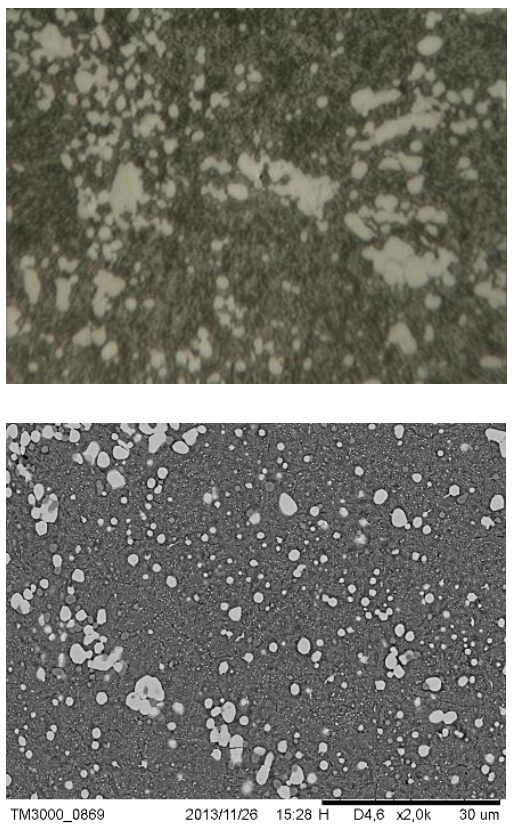

(a)
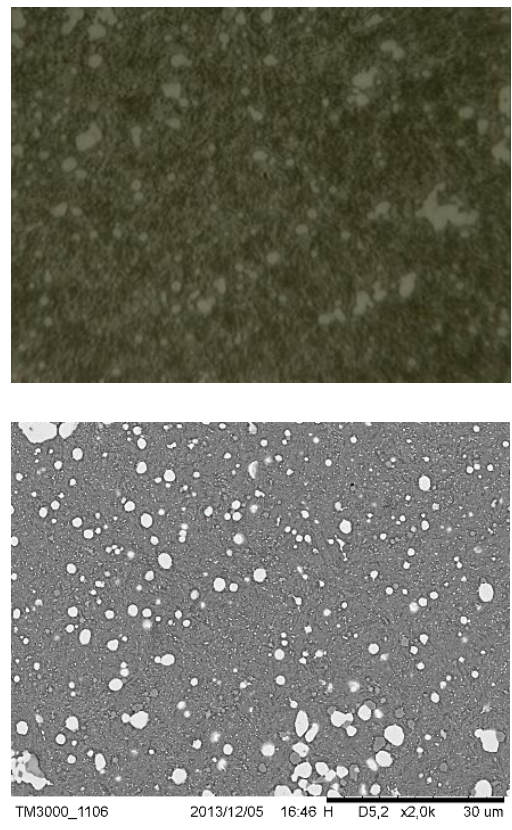

(b)
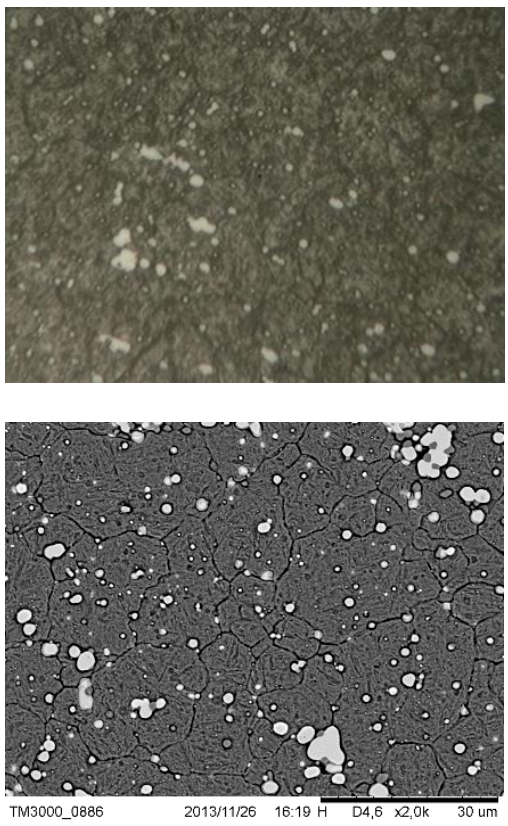

(c)

Figura 2. Fotomicrografias (MO e MEV) do Aço AISI M2 temperados em óleo com $1000^{\circ} \mathrm{C}$ para tempos de encharque distintos: (a) 10 min e (b) 20 min e (c) 60 min.
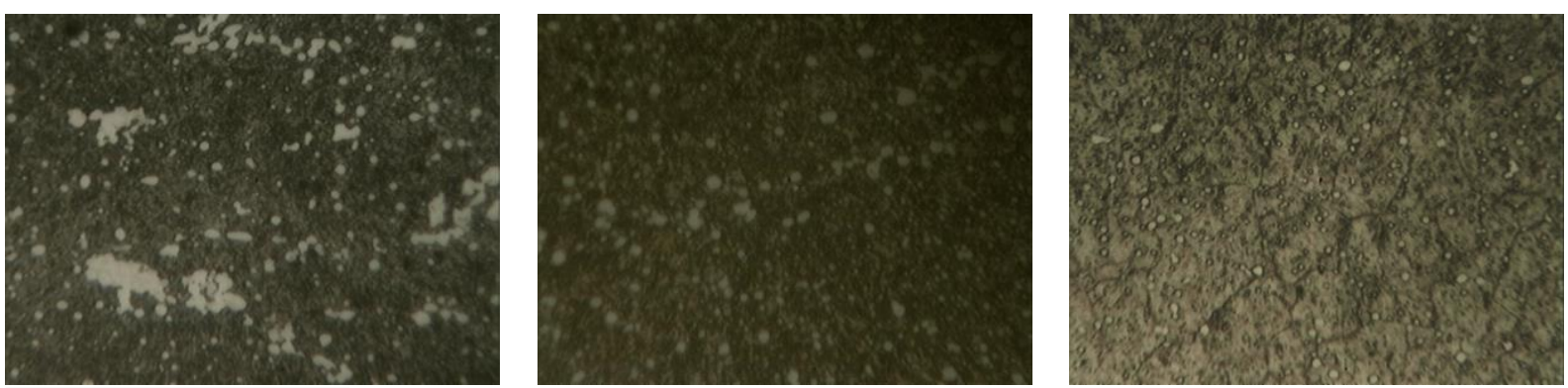

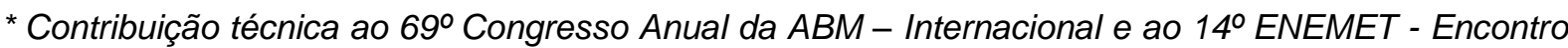
Nacional de Estudantes de Engenharia Metalúrgica, de Materiais e de Minas, 21 a 25 de julho de 2014, São Paulo, SP, Brasil. 


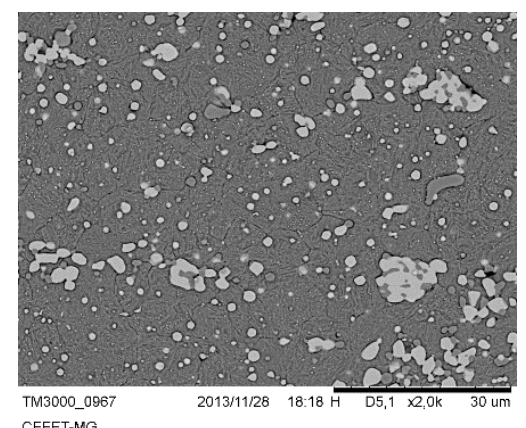

(a)

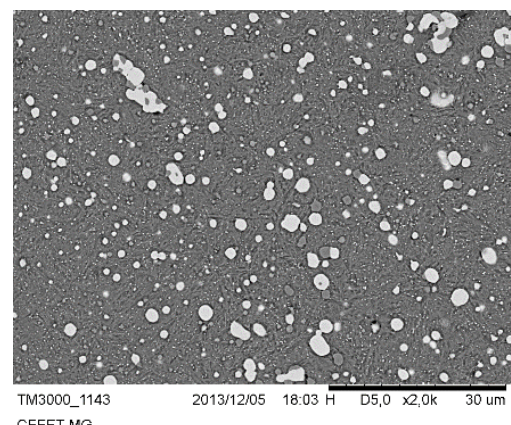

(b)

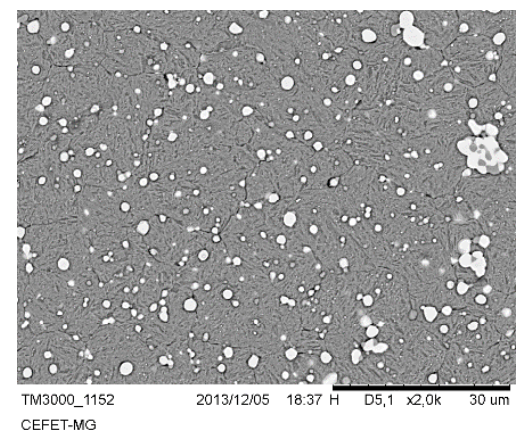

(c)

Figura 3. Fotomicrografias (MO e MEV) do Aço AISI M2 temperados em óleo com $1.100^{\circ} \mathrm{C}$ para tempos de encharque distintos: (a)10 min e (b) 20 min e (c) 60 min.

Nota-se, em geral, uma estrutura com a presença acentuada de carbonetos (pontos mais claros) dispersos sobre uma matriz de martensita, o que vai ao encontro com a literatura [14].
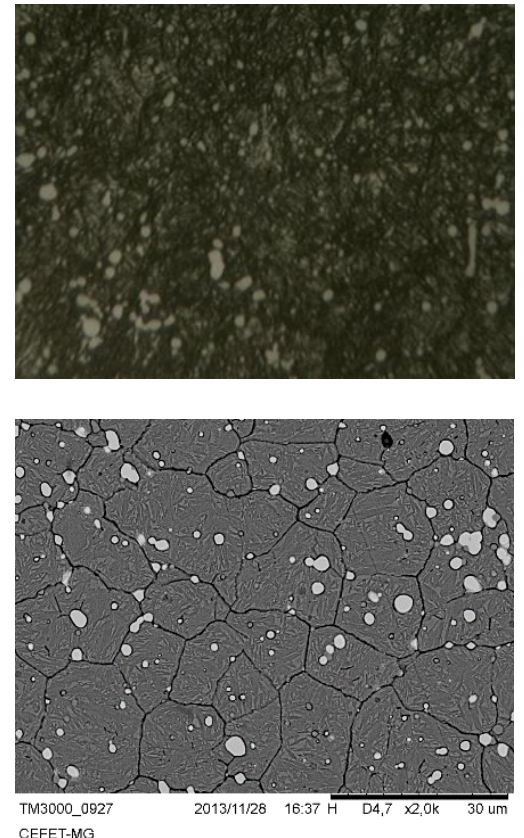

(a)
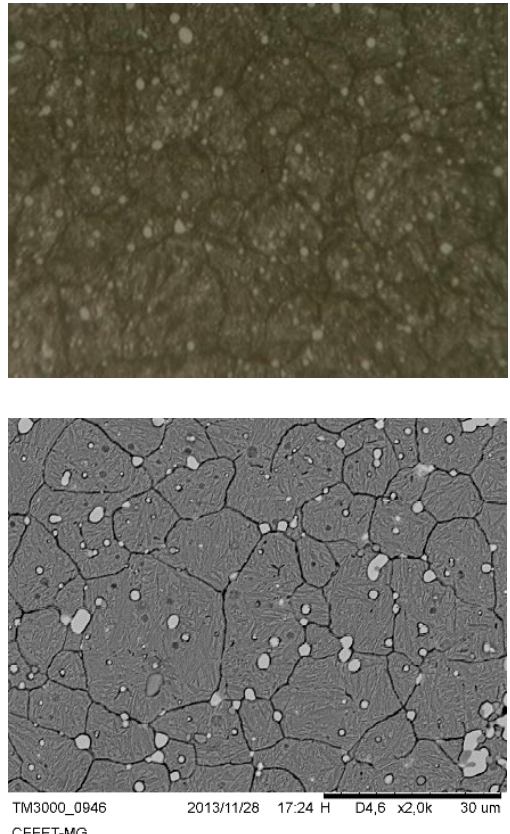

(b)
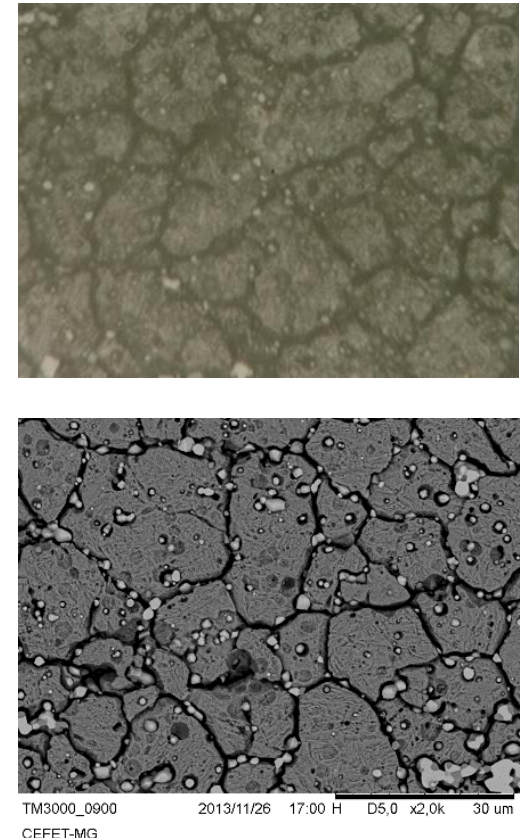

(c)

Figura 4. Fotomicrografias (MO e MEV) do aço AISI M2 temperado em óleo com $1200^{\circ} \mathrm{C}$ para tempos de encharque distintos: (a) 10 min e (b) 20 min e (c) 60 min.

A morfologia dos carbonetos pode ser analisada de maneira mais nítida por meio das imagens de MEV, que demonstram uma estrutura apontada como globular e com variações no seu tamanho. Tal análise é reiterada na literatura, que cita os carbonetos dos tipos $\mathrm{MC} \mathrm{e} \mathrm{M}_{23} \mathrm{C}_{6}$ como característicos de materiais com composição química contendo vanádio, cromo, molibdênio, e tungstênio e com a morfologia apresentada [15].

Em relação ao efeito dos parâmetros de tratamento, observa-se que, em geral, o aumento do tempo de encharque resultou em uma aparente diminuição na quantidade de carbonetos, principalmente para as temperaturas de $1.000^{\circ} \mathrm{C}$ e $1.100^{\circ} \mathrm{C}$.

Considerando a temperatura de austenitização, comportamento similar é verificado, ou seja, de maneira geral, a elevação na temperatura parece levar à redução na

* Contribuição técnica ao $69^{\circ}$ Congresso Anual da ABM - Internacional e ao 14ํㅡㄹ ENEMET - Encontro Nacional de Estudantes de Engenharia Metalúrgica, de Materiais e de Minas, 21 a 25 de julho de 2014, São Paulo, SP, Brasil. 


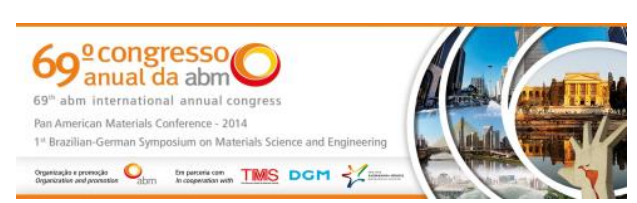

\section{CONCLUSÃO}

De maneira geral, a dureza do aço rápido AISI M2 aumentou com a temperatura de austenitização. Em relação ao tempo de encharque, a elevação nos valores de dureza foi observada de forma clara ao passar de 10 minutos para 20 minutos.

As fotomicrografias revelaram, para as diversas condições de tratamento térmico, a ocorrência de estrutura martensítica e a presença de carbonetos, cuja quantidade pareceu diminuir com o aumento do tempo de encharque e com a elevação da temperatura de austenitização. Os difratogramas corroboraram com os resultados das fotomicrografias, evidenciando ainda a presença de austenita retida.

\section{Agradecimentos}

Os autores agradecem à agência de fomento CAPES (Coordenação de Aperfeiçoamento Pessoal de Nível Superior) pelas bolsas.

\section{REFERÊNCIAS}

1 Herranz G, Romero A, Castro V, Rodrígues G.P. Processing of AISI M2 high speed steel reinforced with vanadium carbide by solar sintering. Materials and Design, 2014; 54: 934-946.

2 Pippel E, Woltersdorf J, Pockl G, Lichtenegger G. Microstructure and nanochemistry of carbide precipitates in high-speed steel S 6-5-2-5. Materiais Characterization, 1999; 43: 41-55.

3 Chiaverini V. Aços e ferros fundidos. 7 ed. São Paulo: Associação Brasileira de Metalurgia e Materiais - ABM, 1996. 599p.

4 Li HY, Luo DF, Lau KH, Yeung CF. Use of low carbon/high ductile high-speed steel to make cutting-tools. Journal of Materials Processing Technology, 2002; 122: 179-184.

5 Coutinho CB. Materiais metálicos para engenharia. Belo Horizonte: UFMG: Fundação Christiano Ottoni, 1992. 405 p.

6 Chaus AS, Dománková M. Precipitation of secondary carbides in M2 high-speed steel modified with Titanium diboride. Journal of Materials Engineering and Performance, 2013; 22: 1402-1420.

7 Zhou XF, Fang F, Li F, Jiang JQ. Mophology and microstructure of $\mathrm{M}_{2} \mathrm{C}$ carbide formed at different cooling rates in AISI M2 high speed steel. Journal of Materials Science, 2011; 46: 1196-1202.

8 Zhang G, Yuan H, Jiao D, Li Z, Liu Z. Microstructure evolution and mechanical properties of T15 high speed steel prepared by twin-atomiser spray forming and thermo-mechanical processing. Materials Science \& Engineering A, 2012; 558: 566571.

9 Xu L, Xing J, Wei S, Zhang Y, Long R. Study on relative wear resistance and wear stability of high-speed steel with high vanadium content. Wear, 2007; 262: 253-261.

10 Suchánek J, Kuklík V. Influence of heat and thermochemical treatment on abrasion resistance of structural and tool steels. Wear, 2009; 267: 2100-2108.

11 Serna MM, Rossi JL. MC complex carbide in AISI M2 high speed steel. Materials Letters, 2009; 63, 2009: 691-693.

12 Bochnowskia W, Leitner H, Major L, Ebner R, Major B. Primary and secondary carbides in high-speed steels after conventional heat treatment and laser modification. Materials Chemistry and Physics, 2003; 81: 503-506.

13 Schmolz, Bikenbach. Rapidur 3343. Catálogo do Fabricante. Disponível em:<http://www.schmolz-bickenbach.com.br/fileadmin/files/schmolz-bickenbach. com.br/documents/Fichas_Tecnicas_novo_formato_dez2011/Acos_rapidos/Rapidur_33 43.pdf>. Acesso em: 23 mar. 2013.

\footnotetext{
* Contribuição técnica ao $69^{\circ}$ Congresso Anual da ABM - Internacional e ao 14ํㅡㄹ ENEMET - Encontro Nacional de Estudantes de Engenharia Metalúrgica, de Materiais e de Minas, 21 a 25 de julho de 2014, São Paulo, SP, Brasil.
} 
14 ASM Handbook. Metallography and microstructures. Ohio: ASM International, 2004. $1184 p$.

15 Vitry V, Nardone S, Breyer JP, Sinnaeve M, Delaunois F. Microstructure of two centrifugal cast high speed steels for hot strip mills aplications. Materials and Design, 2012; 34: 372-378.

16 Akbari A, Mohammadzadeh R, Templier C, Rivieri JP. Effect of the initial microstructure on the plasma nitriding behavior of AISI M2 high speed steel. Surface \& Coatings Technology, 2010; 204: 4114-4120.

17 Dobrzanski LA, Matula G, Varez A, Levenfeled B, Torralba JM. Fabrication methods and heat treatment conditions effect on tribological properties of high speed steels. Journal of Materials Processing Technology, 2004; 157: 324-330.

18 Moon HK, Lee KB, Kwon H. Influences of Co addition and austenitizing temperature on secondary hardening and impact fracture behavior in P/M high speed steels of W-Mo$\mathrm{Cr}-\mathrm{V}(-\mathrm{Co})$ system. Materials Science and Engineering A, 2008; 474: 328-334.

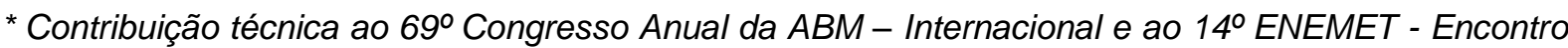
Nacional de Estudantes de Engenharia Metalúrgica, de Materiais e de Minas, 21 a 25 de julho de 2014, São Paulo, SP, Brasil. 